\title{
A review of non-destructive thermography techniques toward structural integrity of bio-composites
}

\begin{abstract}
It is well known those two popular methods of testing; destructive testing based on fracture mechanics and non-destructive testing (NDT) which does not make any damage in the specimen. NDT was first used for military purpose but nowadays it is used widely in many fields such as composite materials, medical purposes, fire safety, laser welding, food safety and quality and characterization of materials. The aim of this paper is to review the recent advancement of thermography non-destructive methods especially in testing a quality of biocomposites materials. The review reveals the advantages and disadvantages of pursuing any of the available methods in NDT on bio composite materials.
\end{abstract}

Keyword: Quality control; Non-destructive testing; Composite materials; Thermal imaging; Infrared camera 